\title{
Criminologie
}

\section{Les projets de lois et de politiques en matière de victimes d'actes criminels}

\section{Micheline Baril}

Volume 21, numéro 2, 1988

Les soins de santé mentale en milieu carcéral

URI : https://id.erudit.org/iderudit/017269ar

DOI : https://doi.org/10.7202/017269ar

Aller au sommaire du numéro

Éditeur(s)

Les Presses de l'Université de Montréal

ISSN

0316-0041 (imprimé)

1492-1367 (numérique)

Découvrir la revue

Citer cet article

Baril, M. (1988). Les projets de lois et de politiques en matière de victimes

d'actes criminels. Criminologie, 21(2), 103-111. https://doi.org/10.7202/017269ar d'utilisation que vous pouvez consulter en ligne.

https://apropos.erudit.org/fr/usagers/politique-dutilisation/ 
La dernière année aura été fertile en projets de toutes sortes concernant les victimes d'actes criminels, tant au Québec que dans le reste du Canada. Des projets de loi ont été déposés, des politiques sont à l'étude, des comités ont été formés, des services d'aide créés.

Rappelons que l'intérêt à l'égard des victimes est né en Europe à la fin des années 40 et qu'il s'est rapidement propagé à l'Amérique du Nord (Pennsylvanie et Québec, en particulier). C'était un intérêt plutôt académique, parfois politique, orienté surtout vers la compréhension du passage à l'acte. Voilà que durant les années 70 , sous l'influence de groupes féministes et l'apport des sondages de victimisation, apparaît une prise de conscience du mécontentement du public à l'égard des systèmes de justice criminelle. Cette prise de conscience favorisa la formation d'associations de défense des droits des victimes, telles que la National Organization for Victims Assistance (NOVA), Mothers Against Drunk Driving (MADD), Victims of Violence Inc., etc. Aux États-Unis, ces mouvements entraînèrent à la fois une prolifération rapide de centres d'aide aux victimes et des changements législatifs importants aux paliers fédéral et des États. Les pays d'Europe s'engagèrent plus tard dans cette voie mais le firent avec une rapidité étonnante sans toutefois utiliser beaucoup les voies législatives.

Le Canada qui, antérieurement, avait fait figure de précurseur adopta une position prudente en matière de législation et de services à l'intention des victimes. Il était pourtant un des trois pays, avec l'Australie et la France, qui avaient contribué activement à la déclaration des principes de justice à l'égard des victimes qui fut adoptée par l'Assemblée des Nations unies en décembre 1985.

En 1987 et 1988, toutefois, le Canada a mis de l'avant de nombreux projets de lois et de politiques. Nous nous attarderons à l'initiative qui semble la plus importante, le projet de loi C-89. En second lieu, nous présenterons le projet de loi québécoise, la seconde législation provinciale après celle du Manitoba. En terminant, nous mentionnerons quelques autres initiatives récentes sur les scènes fédérale et provinciale.

* Professeure à l'École de criminologie de l'Université de Montréal, C.P. 6128, Succ. «A», Montréal (Québec) H3C 3J7 


\section{PROJET DE LOI C-89, LOI MODIFIANT LE CODE CRIMINEL (VICTIMES D'ACTES CRIMINELS)}

Actuellement, en juin 1988, le projet est à l'étude au comité sénatorial permanent des affaires juridiques et constitutionnelles. Il comprend cinq thèmes.

\subsection{LA PROTECTION DE L'IDENTITÉ DES PLAIGNANTS ET TÉMOINS}

Cet amendement vise à protéger davantage les victimes mineures et les victimes et témoins d'agressions sexuelles et d'extorsion. On peut croire que la protection de l'anonymat épargnerait des souffrances aux victimes tout en facilitant leur collaboration au processus de justice. On a cependant noté des objections à cet amendement en vertu de la liberté de presse et du droit de l'accusé à un procès public. Monsieur Robert Wakefield, ancien président de l'Association des procureurs de la défense d'Ottawa, plaide la liberté fondamentale de la presse et la nécessité d'une justice à ciel ouvert. Il ajoute cependant que : «C'est au juge d'établir si une raison prépondérante manifeste exige d'interdire la divulgation des noms des parties».

\subsection{LE RETOUR DES BIENS VOLÉS OU CONFISQUÉS}

Ces amendements semblent peu contestés. De fait, ils entérinent des pratiques déjà en cours dans plusieurs districts judiciaires à travers le Canada. Jadis, les biens volés et recouvrés et ceux qui avaient été saisis comme éléments de preuve pouvaient être gardés jusqu'à la fin des procédures judiciaires. L'État conservait à grands frais des pièces à conviction rarement utilisées en cour alors que leurs propriétaires subissaient une double perte (Baril et al., 1984). Le recours à la preuve photographique, en usage depuis près de dix ans dans certains districts, assiste les victimes tout en ménageant les deniers publics.

\subsection{LE DÉDOMMAGEMENT PAR L'AUTEUR DU PRÉJUDICE}

C'est l'amendement le plus important, à mon avis. Le projet de loi C-89 favorise le dédommagement et lui accorde même priorité sur l'amende. Le tribunal serait tenu d'examiner les possibilités de dédommagement et de motiver sa décision s'il ne statue pas en ce sens. Cependant, selon le projet C-89, il semble que le dédommagement doive être imposé «en sus de toute autre peine». Cette stipulation soulève beaucoup d'objections dans les milieux juridiques et criminologiques. 
Les juristes soulèvent surtout les questions constitutionnelles! La réparation est du ressort civil, donc provincial. Si le dédommagement de la part de l'auteur des préjudices était reconnu comme une peine et non comme une réparation, le gouvernement fédéral pourrait statuer et admettre que le dédommagement constitue une peine unique et non accessoire. Telle décision entraînerait des changements majeurs dans la philosophie du droit pénal.

Les criminologues redoutent une inflation des peines et une discrimination à l'égard des moins fortunés. Cette crainte a été exprimée dans le mémoire soumis par Baril, Landreville, Normandeau (1987).

\subsection{LA DÉCLARATION DE LA VICTIME AU TRIBUNAL}

Le projet de loi C-89 prévoit que la victime pourra être entendue au moment du prononcé de la sentence pour faire connaître son préjudice au tribunal. Dans les pays de droit commun, c'est un moyen de favoriser l'accès à la justice pour les victimes. Déjà, six districts judiciaires canadiens ont mis sur pied des projets expérimentaux de déclaration de la victime (Giliberti, 1987).

À Montréal, le projet a débuté en octobre 1987, sous la responsabilité de l'Association québécoise plaidoyer-victimes. Les principes qui le guident sont :

- la reconnaissance de l'égalité de tous devant la justice, victimes et accusés;

- le respect du besoin d'être entendues qu'éprouvent les victimes;

- la nécessité de rendre justice à partir de la connaissance la plus complète possible des éléments pertinents.

Il vise donc à la fois des fins humanitaires et des objectifs d'équité.

Plusieurs juristes ont fait savoir à la Chambre des communes et au Sénat leurs réticences face à la déclaration de la victime, réticences basées surtout sur la crainte d'introduire, dans la détermination de la peine, un élément de vengeance. Ces appréhensions ne sauraient être prises à la légère. Cependant, à Montréal du moins, ni les victimes ni les juges ont penché vers la rétribution. Dans le district judiciaire de Montréal, environ $50 \%$ des victimes ont déposé une déclaration et très peu d'entre elles ont émis une opinion sur la sentence. Les rapports de recherche dans les six districts judiciaires canadiens seront bientôt disponibles et permettront de mieux connaître l'impact de cette disposition. 
Soulignons enfin que la déclaration de la victime, le «victim impact statement» a été adoptée par la grande majorité des États américains (NOVA, 1985).

\subsection{LA SURAMENDE}

Cette disposition prévoit que toute personne condamnée en vertu du Code criminel, de la Loi des aliments et drogues ou de la Loi sur les stupéfiants, devrait, en sus de toute autre peine, verser une suramende compensatoire qui serait affectée à l'aide aux victimes.

Voilà une proposition qui est loin de susciter un consensus. Pourquoi imposer une suramende aux infracteurs qui n'ont pas lésé d'autres individus? Quelle est la valeur éducative d'une suramende automatique et anonyme? Le cas échéant, comment déterminer équitablement les taux ou montants de suramendes? À quelles fins précises seraient affectées les sommes perçues?

\subsection{EN CONCLUSION}

Le projet de loi C-89 suggère des changements majeurs dans le droit pénal canadien. J'aimerais citer ici le mémoire soumis en décembre 1987 par l'École de criminologie et le Centre international de criminologie comparée de l'Université de Montréal (Baril, Landreville, Normandeau).

Pour peu, on parlerait d'un projet révolutionnaire. C'est la première fois que le législateur canadien prend en considération les intérêts de tous les justiciables et plus particulièrement ceux de l'ensemble des victimes d'actes criminels, s'orientant ainsi vers une justice sociale et non plus strictement pénale. Ce faisant, il répond aux propositions de nombreuses commissions d'étude (Commission de réforme du droit, Commission sur la détermination de la peine, groupe d'étude fédéral-provincial sur les victimes d'actes criminels, ...); il légalise et encourage des pratiques déjà en cours; il poursuit une orientation amorcée par d'autres législations ou projets de loi (C-19, C-127, C-15,...); enfin et surtout, il reconnaît une réalité sociale actuelle et il est conforme à la déclaration acceptée par l'ONU en décembre 1985.

Nous souscrivons à la philosophie sous-jacente au projet de loi C-89 qui, si nous l'avons bien comprise, met en relief la réparation du préjudice comme un des objectifs de la justice pénale. Cependant, C-89 est loin d'être limpide dans ses visées. En effet, le projet n'énonce pas d'objectifs et, du fait même, il laisse prise à des interprétations qui ne concorderont pas nécessairement avec l'intention du législateur. 


\section{LOI 8, LOI SUR L'AIDE AUX VICTIMES D'ACTES CRIMINELS}

Le ministre de la Justice du Québec a déposé ce projet de loi à l'Assemblée nationale en mai dernier; l'Assemblée l'a adopté et la Loi sera bientôt en vigueur. C'est une loi qui va au-delà des autres législations fédérales et provinciales en ce qui concerne les «droits» des victimes. Essentiellement, elle reconnaît des droits aux victimes, crée un Bureau provincial d'aide aux victimes et prévoit un financement des services locaux ou régionaux d'aide.

La loi a d'abord le mérite de définir les victimes:

Dans la présente loi, est considérée comme une victime d'un acte criminel toute personne physique qui, à l'occasion d'un acte criminel commis au Québec, subit une atteinte à son intégrité physique ou psychologique ou une perte matérielle, que l'auteur de cet acte criminel soit ou non identifié, arrêté, poursuivi ou reconnu coupable.

Sont également considérées comme des victimes ses proches et ses personnes à charge.

\subsection{LES DROITS ET LES RESPONSABILITÉS DES VICTIMES}

Avec plusieurs caveat («dans la mesure prévue par la loi», «aussi complètement que possible», «compte tenu des ressources disponibles»), les victimes se verraient accorder des droits à l'information, à la participation, à la protection et à la réparation. Ces dispositions appuient et renforcent les mesures prévues par le projet C-89 et par la déclaration de l'ONU.

Ce chapitre du projet de loi est si intéressant qu'il doit être cité intégralement.

Art. 2. La victime d'un acte criminel a droit d'être traitée avec courtoisie, équité, compréhension et dans le respect de sa dignité et de sa vie privée.

Art. 3. La victime a droit, dans la mesure prévue par la loi :

1. de recevoir une indemnité raisonnable pour les frais encourus en vue de rendre témoignage;

2. de recevoir, de façon prompte et équitable, réparation ou indemnisation des dommages subis;

3 . de se voir restituer les biens saisis dans les meilleurs délais lorsque leur rétention n'est plus nécessaire pour les fins de la justice;

4. de voir ses points de vue et ses préoccupations présentés et examinés aux phases appropriées de toute procédure judiciaire, lorsque son intérêt personnel est en cause. 
Art. 4. La victime a droit, aussi complètement que possible:

1. d'être informée de ses droits et des recours dont elle dispose;

2. d'être informée de son rôle dans le cadre du processus pénal, de sa participation dans la procédure judiciaire et, lorsqu'elle en fait la demande, de l'état et de l'issue de celle-ci;

3. d'être informée de l'existence de services de santé et de services sociaux de même que de tout autre service d'aide ou de prévention propres à lui assurer l'assistance médicale, psychologique et sociale requise.

Art. 5. Lorsqu'elle en fait la demande, la victime a droit, dans la mesure du possible et compte tenu de l'intérêt public, d'être informée de l'état et de l'issue de l'enquête.

Art. 6. Compte tenu des ressources disponibles, la victime a droit: 1. de recevoir l'assistance médicale, psychologique et sociale que requiert son état ainsi que les autres services d'aide appropriés à ses besoins en matière d'accueil, d'assistance et de référence aux autres services les plus aptes à lui venir en aide;

2. de bénéficier de mesures de protection contre les manœuvres d'intimidation et les représailles.

\subsection{LE BUREAU D'AIDE AUX VICTIMES D'ACTES CRIMINELS}

La loi prévoit l'institution d'un Bureau provincial qui devra veiller à la concertation et à la coordination des divers organismes ceuvrant dans le domaine de l'aide aux victimes. Elle doit également favoriser l'implantation et le maintien des services de même que leur financement.

Voilà une autre initiative intéressante. On déplore toutefois qu'un tel Bureau ne soit composé que de fonctionnaires (nombre indéterminé). Il serait souhaitable que tous les groupes concernés (victimes, centres d'aide, procureur, police, ...) soient représentés au Bureau.

\subsection{LE FONDS D'AIDE AUX VICTIMES}

Ce chapitre du projet de loi prévoit de quelles sources proviendront les sommes versées au fonds d'aide et à quelles fins sera affecté ce fonds. Il faut noter que le financement dépend des contributions fédérales, notamment des suramendes compensatoires prévues au projet de loi C-89. Le fonds contribuera au service d'aide aux victimes, à la recherche, au programme d'information, de sensibilisation et de formation. 


\section{QUELQUES INITIATIVES INTÉRESSANTES}

Il importe de souligner que le Canada vient d'adopter une déclaration de principes touchant la justice pour les victimes d'actes criminels (l'énoncé est reproduit à la fin de cet article) et qu'une association nationale de défense des droits des victimes est en voie de formation.

Au Québec, c'est dans le domaine de la violence intrafamiliale, conjugale surtout, que les progrès sont les plus rapides. Une politique provinciale a été élaborée et a inspiré la politique du service de police de la Communauté urbaine de Montréal qui se compare maintenant favorablement avec les réalisation des autres grands corps de police canadiens.

Du côté des abus exercés à l'égard des personnes âgées, un comité formé par la ministre de la Santé et des Services sociaux doit formuler très bientôt des recommandations quant à la prévention des abus et l'intervention auprès des victimes.

\section{CONCLUSION}

Au yeux de ceux qui les préconisent les progrès sont lents. Avec le recul de l'histoire, ils apparaîtront probablement rapides. C'est toute une conception de la justice qui se transforme sous nos yeux comme en témoignent les projets de lois que nous venons de décrire.

En 1980, quand les divers paliers de gouvernement ont commencé à s'intéresser à la violence conjugale, ils croyaient qu'une période de cinq ans serait nécessaire pour contrôler le problème. Cependant, il a fallu ces cinq années pour mieux comprendre le phénomène et commencer à élaborer des politiques. Il en faudra beaucoup plus pour trouver les solutions. Quant aux autres types de violence et de victimes, nous en sommes à nos tous premiers pas. Au moins deux excellentes questions sont posées : Qu'est-ce que la justice? Justice pour qui?

\section{ÉNONCÉ DE PRINCIPES POUR LES VICTIMES D'ACTES CRIMINELS}

1. Les victimes devraient être traitées avec courtoisie, compassion et respect pour leur dignité et leur vie privée et devraient souffrir le moins possible du dérangement inévitable qu'entraîne leur participation au système de justice pénale.

2. Des procédures officielles et officieuses devraient être mises en place pour permettre de réparer rapidement et équitablement le tort que les victimes ont subi. 
3. Les victimes devraient être informées de la réparation qu'elles peuvent obtenir et des mesures à prendre à cette fin.

4. Les victimes devraient être informées du rôle qu'elles joueront dans le cadre du procès, de la date des audiences, du déroulement de l'affaire et de la décision finale du tribunal.

5. On devrait déterminer quelles sont les opinions et les préoccupations des victimes et leur fournir l'aide dont elles ont besoin pendant tout le procès.

6. Lorque les intérêts personnels de la victime sont en cause, ses opinions et ses préoccupations devraient être portées à l'attention du tribunal si les règles de droit et de procédure en matière pénale le permettent.

7. On devrait, au besoin, prendre les mesures nécessaires pour assurer la sécurité des victimes et de leur familles et les protéger contre les menaces et les représailles.

8. Les personnes travaillant dans le domaine de la justice pénale devraient bénéficier d'une meilleure formation de façon à être sensibilisées aux besoins et aux préoccupations des victimes, et des lignes directrices devraient, au besoin, être élaborées à cette fin.

9. Les victimes devraient être informées des services de santé et des services sociaux qui sont offerts de même que de toute autre forme d'aide qu'elles peuvent obtenir de façon qu'elles puissent continuer de bénéficier de l'aide médicale, psychologique et sociale dont elles ont besoin grâce aux programmes et aux services en place.

10. Les victimes devraient dénoncer le crime et collaborer avec les autorités chargées de l'applicaiton de la loi.

\section{RÉFÉRENCES}

BARIL, M. (1987), «Une première canadienne en matière de victimes d'actes criminels", Actualités-Justice, 4,1, p. 8.

BARIL, M., P. LANDREVILLE et A. NORMANDEAU (1987), Mémoire soumis au Comité législatif sur le projet de loi C-89, École de criminologie et Centre international de criminologie comparée, Université de Montréal.

BARIL, M., S. DURAND, M.M. COUSINEAU et S. GRAVEL (1984), Mais nous, les témoins .... Montréal, Centre international de criminologie comparée, Université de Montréal. 
CANADA (1988), Délibérations du Comité sénatorial permanent des affaires juridiques et constitutionnelles, 31 maj 1988 , fasc. 78 , p. 7 .

CANADA (1988), Énoncé de principes pour les victimes d'actes criminels, Ottawa, ministère de la Justice.

GILIBERT1, C. (1987), Victim Impact Statements in Canada : Proposed Models and Evaluation Designs, Ottawa, ministère de la Justice.

NOVA (1985), Victims Rights and Services : A Legislative Directory, Washington, U.S. Department of Justice.

ORGANISATION DES NATIONS UNIES (1985), Déclaration des principes fondamentaux de justice relatifs aux victimes de la criminalité et aux victimes d'abus de pouvoir, A/RES/40/34, 11 décembre 1985. 